PROCEEDINGS OF THE

AMERICAN MATHEMATICAL SOCIETY

Volume 131, Number 9, Pages 2715-2720

S 0002-9939(03)07140-5

Article electronically published on April 23, 2003

\title{
DIFFERENTIABLE CONJUGACY OF THE POINCARÉ TYPE VECTOR FIELDS ON $\mathbf{R}^{3}$
}

\author{
JIAZHONG YANG
}

(Communicated by Jozef Dodziuk)

\begin{abstract}
We prove that on $\mathbf{R}^{3}$, except for those germs of vector fields whose linear parts are conjugated to $\lambda x \partial / \partial x+\lambda y \partial / \partial y+2 \lambda z \partial / \partial z$, any two Poincaré type vector fields are at least $C^{3}$ conjugated to each other provided their linear approximations have the same eigenvalues and the nonlinear parts are generic.
\end{abstract}

\section{Introduction AND THE MAIN RESUlt}

Consider a smooth germ of a vector field at a singular point 0 given by an ordinary differential equation

$$
\dot{x}=\frac{d x}{d t}=A x+\cdots, \quad x \in \mathbf{R}^{n}, t \in \mathbf{R},
$$

where $A$ is an $n \times n$ matrix and the dots denote the higher order terms. We denote by $\lambda=\left(\lambda_{1}, \ldots, \lambda_{n}\right)$ the eigenvalues of the matrix $A$. If $\lambda$ does not belong to the imaginary axis (i.e., the real part of $\lambda_{j}$ is different from 0 for all $j$ ), then conventionally we call system (1) a hyperbolic vector field at the equilibrium point. Moreover, the tuple $\lambda$ is said to belong to the Poincaré domain if the convex hull of the eigenvalues $\lambda_{1}, \ldots, \lambda_{n}$ in the complex plane does not contain the origin. Correspondingly, vector field (1) is said to be of Poincaré type if $\lambda$ belongs to the Poincaré domain. It is clear that any Poincaré type vector field is hyperbolic.

The present paper is devoted to establishing finitely smooth equivalence of the Poincaré type vector fields on $\mathbf{R}^{3}$. The motivation is based on the distinguished Hartman-Grobman theorem which says that any hyperbolic vector field is topologically linearizable. In other words, the Hartman-Grobman theorem says that the linear approximations of hyperbolic vector fields totally determine their $C^{0}$ classification. Note that the conjugating homeomorphism generally fails to be a diffeomorphism as shown in the example (see [4]): $\dot{x}=2 x, \dot{y}=y+x z, \dot{z}=-z$. This is a non-Poincaré type hyperbolic vector field. For the Poincaré type vector fields, however, we find that the linear approximations generally imply more information than topological linearization and thus yield stronger (than $C^{0}$ ) classifications, especially if we are allowed to preserve some nonlinear terms. In the 2-dimensional case, the situation is obvious since any Poincaré type vector field

Received by the editors August 20, 2000.

2000 Mathematics Subject Classification. Primary 34K17, 37C15.

Key words and phrases. Conjugacy, Poincaré type vector fields, moduli, normal form, resonance. 
admits at most one resonant relation. In the 3 -dimensional case, the situation is much less trivial and it involves many cases which can have quite complicated resonant relations. In this paper we shall primarily study the 3-dimensional case. More precisely, we shall prove the following result.

Main Theorem. In the set of all smooth Poincaré type vector fields on $\mathbf{R}^{3}$, except for those systems whose linear approximations are conjugated to

$$
\dot{x}=\lambda x, \quad \dot{y}=\lambda y, \quad \dot{z}=2 \lambda z,
$$

any two vector fields having the same eigenvalues and generic nonlinear parts are at least $C^{3}$ conjugated.

Remark 1. Up to smooth $\left(C^{\infty}\right)$ conjugacy, there are only two kinds of vector fields having linear approximation (2):

$$
\dot{x}=\lambda x, \quad \dot{y}=\lambda y, \quad \dot{z}=2 \lambda z+x^{2} \pm y^{2} .
$$

It is easy to prove that vector fields corresponding to the case " + " and to the case "_" are only $C^{1}$ conjugated.

We point out that in many cases the theorem remains valid in the $C^{\infty}$ setting.

Example 1. All vector fields having eigenvalues $(1,2,2 n)$ in the generic case are smoothly conjugated to each other, and they are conjugated to

$$
\dot{x}=x, \quad \dot{y}=2 y+x^{2}, \quad \dot{z}=2 n z+y^{n} .
$$

\section{Preliminaries}

The classification of the Poincare type vector fields mentioned above is related to their simplest resonant normal forms. To describe it, we first introduce below some definitions and recall some known facts.

Definition 2.1. Let $X$ be a smooth vector field on $\mathbf{R}^{n}$ having the form (1), where the matrix $A$ has eigenvalues $\left(\lambda_{1}, \ldots, \lambda_{n}\right)$. Then any relation

$$
\lambda_{j}=k_{1} \lambda_{1}+\cdots+k_{n} \lambda_{n}, \quad k_{s} \in \mathbf{Z}_{+} \cup\{0\}, \quad k_{1}+\cdots+k_{n} \geq 2,
$$

is called a resonant relation having an order $k_{1}+\cdots+k_{n}$, and the term $x_{1}^{k_{1}} \cdots x_{n}^{k_{n}} \frac{\partial}{\partial x_{j}}$ is called a resonant monomial.

The vector field (11) is said to be in resonant (Poincaré-Dulac) normal form if the matrix $A$ has been normalized to the Jordan normal form and its nonlinear part consists of resonant monomials only.

Recall that any smooth hyperbolic vector field can be smoothly reduced to its resonant normal form. This is due to the Sternberg-Chen theorem [6, 3] (the formal setting of the statement is due to the Poincare-Dulac theorem; see [1]). Note also that any tuple belonging to the Poincaré domain admits a finite number of resonant relations (see [5]). Consequently, the resonant normal form of any Poincaré type vector field is polynomial.

The Poincaré-Dulac normal form is based on the resonant relations of the linear part of a vector field and generally admits further simplification. Indeed, a Poincaré type vector field, under certain genericity conditions on the nonlinear terms, can be reduced to the simplest resonant normal form. If a vector field is finitely determined, then the simplest resonant normal form is a resonant normal form that is of the lowest possible order and contains the minimal number of parameters. When the 
simplest normal form is a polynomial, we call the degree of the polynomial the index of finite determinacy and the number of parameters the moduli. It is clear that the index of finite determinacy and the moduli are invariants of a system. For more details about the finitely determined vector fields, the normalization of vector fields and the simplest normal form, please refer to [5, 2, 7, 8,

Note that except for the Poincaré type vector fields there exists a class of vector fields which admit infinitely many resonant relations but they are still reducible to a polynomial. Such vector fields, however, always have moduli in their simplest normal forms.

2.1. The classification of 3-dimensional Poincaré type tuples. Let $\lambda=$ $\left(\lambda_{1}, \lambda_{2}, \lambda_{3}\right)$ be a 3 -tuple belonging to the Poincaré domain. Then one can prove that the following list exhausts all the possibilities of $\lambda$.

Case 1. There is no resonant relation between $\lambda$.

Case 2. There is only one resonant relation which up to enumeration of eigenvalues has the form $\left(\lambda_{1}, \lambda_{2}, k_{1} \lambda_{1}+k_{2} \lambda_{2}\right)$, where $k_{1}$ and $k_{2}$ are nonnegative integers with their sum bigger than 1 . For example, the tuples $(1, \sqrt{2}, 1+\sqrt{2})$ and $(2,3,7)$ belong to this case.

Case 3. There is a parameter $\lambda_{0} \neq 0$ such that the eigenvalues are of the form $(p, q, r) \lambda_{0}$, where $p, q$, and $r$ are integers satisfying $1<p<q<r,(p, q)=1$, and $r$ has more than one resonant combination $r=l_{1} p+l_{2} q$. For example, $\lambda=(2,3,12)$.

Case 4. The eigenvalues are multiple and have the form $\lambda=(1,1, p) \lambda_{0}$ for some integer $p \geq 2$. We shall distinguish three subcases:

Case 4.1. The matrix $A$ is nondiagonalizable.

Case 4.2. The matrix $A$ is diagonalizable and $p=2,3$.

Case 4.3. The matrix $A$ is diagonalizable and $p \geq 4$.

Case 5. The eigenvalues are multiple and have the form $\lambda=(1, p, p) \lambda_{0}$ for some integer $p>1$.

Case 6 . The eigenvalues have the form $\lambda=(1, p, q) \lambda_{0}$, where $2 \leq p<q$, and $p$ and $q$ are integers.

2.2. The moduli-free normal forms. If, apart from the eigenvalues, a simplest normal form of a vector field contains no other parameters distinguishing the $i$-jet of the vector field, where $i$ is the index of finite determinacy, then the vector field is moduli-free. Moduli-free vector fields have important dynamical properties. Yet, in general, it is still an open question to know what kind of eigenvalues of a given hyperbolic vector field yields a moduli-free normal form. In the 3-dimensional case, however, the question is known due to the following lemma.

Lemma 2.1. If $X$ is a vector field whose eigenvalues do not belong to Case 4.3 or to Case 3 where there are more than two resonant relations, then $X$ is moduli-free.

Note that in Case 3 if there are exactly two resonant relations, then the corresponding vector fields are also moduli-free. We shall consider this case in more detail in the next subsection.

Below we give a list of the moduli-free normal forms (see [8]).

Case 1:

$$
\dot{x}=\lambda_{1} x, \quad \dot{y}=\lambda_{2} y, \quad \dot{z}=\lambda_{3} z .
$$


Case 2:

$$
\dot{x}=\lambda_{1} x, \quad \dot{y}=\lambda_{2} y, \quad \dot{z}=\lambda_{3} z+x^{k_{1}} y^{k_{2}} .
$$

Case 4.1:

$$
\dot{x}=\lambda x, \quad \dot{y}=\lambda y+x, \quad \dot{z}=p \lambda z+y^{p} .
$$

Case 4.2:

$$
\begin{array}{lll}
p=2: & \dot{x}=\lambda x, \quad \dot{y}=\lambda y, \quad \dot{z}=2 \lambda z+x^{2} \pm y^{2} . \\
p=3: & \dot{x}=\lambda x, \quad \dot{y}=\lambda y, \quad \dot{z}=3 \lambda z+x^{3}+y^{3} .
\end{array}
$$

Case 5:

$$
\dot{x}=\lambda x, \quad \dot{y}=p \lambda y+\delta z, \quad \dot{z}=p \lambda z+x^{p}, \quad \delta=0,1 .
$$

Case 6:

$$
\dot{x}=\lambda x, \quad \dot{y}=p \lambda y+x^{p}, \quad \dot{z}=q \lambda z+x^{q-s p} y^{s}, \quad s=[q / p] .
$$

2.3. Case 3. As to Case 3, we shall prove the following.

Lemma 2.2. Let $X$ have eigenvalues $(p, q, r) \lambda_{0}$ as described in Case 3.

(i) If the tuple $(p, q, r)$ admits exactly two resonant relations

$$
r=l_{1} p+l_{2} q=l_{1}^{\prime} p+l_{2}^{\prime} q,
$$

then the simplest normal form of $X$ is moduli-free and is given by

$$
\dot{x}=p \lambda_{0} x, \quad \dot{y}=q \lambda_{0} y, \quad \dot{z}=r \lambda_{0} z+x^{l_{1}} y^{l_{2}}+x^{l_{1}^{\prime}} y^{l^{\prime}} .
$$

(ii) If $r$ has more than two resonant combinations, then $r \geq 2 p q$.

Remark 2. The number $r$ having exactly two (resp. $k$ ) resonant combinations can be expressed in terms of $p$ and $q$ explicitly: according to the well-known fact in number theory, they are given by $2 p q-p-q+1 \leq r \leq 3 p q-p-q$ and $r \neq 2 p q+l_{1} p+l_{2} q$, where $l_{1}, l_{2}$ are nonnegative integers (resp. $k p q-p-q+1 \leq r \leq(k+1) p q-p-q$ and $\left.r \neq k p q+l_{1} p+l_{2} q\right)$.

Proof. (i) The resonant normal form in this case is

$$
\dot{x}=p \lambda_{0} x, \quad \dot{y}=q \lambda_{0} y, \quad \dot{z}=r \lambda_{0} z+a x^{l_{1}} y^{l_{2}}+b x^{l_{1}^{\prime}} y^{l_{2}^{\prime}},
$$

where $a$ and $b$ are parameters. We only need to show that both $a$ and $b$ can be scaled to 1 , provided they are different from 0 (they are the genericity conditions).

The problem is equivalent to showing the existence of nonzero parameters $s_{1}, s_{2}$ and $s_{3}$ such that under the linear scalings $x \rightarrow s_{1} x, y \rightarrow s_{2} y$ and $z \rightarrow s_{3} z$, the following equalities hold:

$$
a s_{1}^{l_{1}} s_{2}^{l_{2}}=s_{3}, \quad b s_{1}^{l_{1}^{\prime}} s_{2}^{l_{2}^{\prime}}=s_{3} .
$$

The first equality of (13) can always be satisfied as one takes $s_{3}=a s_{1}^{l_{1}} s_{2}^{l_{2}}$. To show the second equality, one needs to prove the existence of $s_{1}$ and $s_{2}$ such that $s_{1}^{l_{1}-l_{1}^{\prime}} s_{2}^{l_{2}-l_{2}^{\prime}}=b / a$. If $a b>0$, then it is done. In fact, one can take, for example, $s_{1}=1, s_{2}=(b / a)^{1 /\left(l_{2}-l_{2}^{\prime}\right)}$. If $a b<0$, we shall show that at least one of $l_{1}-l_{1}^{\prime}$ and $l_{2}-l_{2}^{\prime}$ is odd.

Assume, without loss of generality, that in the resonant relations (11) $l_{1}>l_{1}^{\prime}$. Then $l_{2}<l_{2}^{\prime}$. If both $l_{1}-l_{1}^{\prime}$ and $l_{2}-l_{2}^{\prime}$ are even, i.e., $l_{1}-l_{1}^{\prime}=2 m$ and $l_{2}^{\prime}-l_{2}=2 n$ for some positive integers $m$ and $n$, then $l_{1}-m=l_{1}^{\prime}+m$ and $l_{2}^{\prime}-n=l_{2}+n$. This 
indicates that there exists another resonant relation $r=\left(l_{1}^{\prime}+m\right) \cdot p+\left(l_{2}+n\right) \cdot q$ different from the two given relations, which yields a contradiction.

Without loss of generality, assume that $l_{1}-l_{1}^{\prime}$ is odd and take $s_{1}=-1$. Then the second equality reads as $s_{2}^{l_{2}-l_{2}^{\prime}}=-b / a$, which is always solvable with respect to $s_{2}$ since $-b / a$ is positive.

We leave the proof of (ii) to the reader as an easy exercise.

Note that in Case 3 one has $2 \leq p<q$. Therefore the lemma has the following corollary.

Corollary 2.1. In Case 3, if $r$ has more than two resonant relations, then the order of each resonant relation is no less than $2 p$.

2.4. The linearization of vector fields. Generally speaking, a hyperbolic vector field with a resonant linear part can be linearized by a $C^{k}$ change of coordinates, but $k$ is less than the lowest order of the resonant relations. The following lemma can be derived directly from the Samovol theorem on the linearization of vector fields (see 1], p. 68, and the references cited therein).

Lemma 2.3 (Samovol). Any vector field with eigenvalues $(1,1, p) \lambda_{0}, p \geq 2$, can be $C^{p-1}$ linearized.

Any vector field with eigenvalues $\left(p, q, l_{1} p+l_{2} q\right) \lambda_{0}$, where $(p, q)=1$ and $l_{1}$ and $l_{2}$ are nonnegative integers with their sum bigger than 1 , can be $C^{l_{1}+l_{2}-1}$ linearized.

\section{Proof of the Main Theorem}

To prove the theorem, we only need to show its validity in all the cases listed in the last section.

Given two Poincaré type vector fields $X$ and $Y$ having the same tuple of eigenvalues $\lambda$, if $\lambda$ has the type as specified in Case 1 (resp. Case 2, Case 4.1, Case 4.2 with $p=3$, Case 5, Case 6), then, according to Lemma 2.1, both $X$ and $Y$ are smoothly conjugated to (4) (resp., (5), (6) , (8), (9), (10) ). Therefore they are smoothly conjugated to each other.

It remains to prove the validity of the theorem in Case 4.3 and in Case 3.

In these cases, we can apply Lemma 2.2. More exactly, in Case 3, if $r<2 p q$ then the eigenvalues admit at most two resonant relations, therefore $X$ and $Y$ can be reduced to the same normal form (12), consequently, they are smoothly conjugated to each other. On the other hand, if $\lambda$ admits more than two resonant relations, then generally $X$ and $Y$ are not smoothly equivalent to each other, since in this case the simplest resonant normal form contains moduli. However, according to Lemma 2.2 this can happen only when $r \geq 2 p q$. Thus, due to Corollary 2.1, all the resonant relations have orders no less than $2 p$. Following Lemma 2.3, we know that both $X$ and $Y$ are at least $C^{2 p-1}$ linearizable, therefore they are at least $C^{3}$ conjugated, recalling that $p \geq 2$.

As to Case 4.3 , we can apply similar arguments. In fact, any vector field $X$ can be reduced to the normal forms

$$
\dot{x}=\lambda_{0} x, \quad \dot{y}=\lambda_{0} y, \quad \dot{z}=p \lambda_{0} z+P(x, y),
$$

where $P$ is a homogeneous polynomial of degree $p$.

It is clear that generally for a different vector field $\tilde{X}$, the corresponding polynomial $\tilde{P}$ in the above normal form is not equivalent to $P$. Namely, if $p \geq 4$ in Case 
4.3, then the simplest normal form is not moduli-free. However, due to Lemma 2.3 such a vector field is $C^{p-1}$ linearizable. Therefore, any two vector fields are at least $C^{3}$ conjugated to each other, and both are reducible to the linearized system.

We have proved the theorem.

\section{REFERENCES}

[1] V. Arnol'd, Yu. Ilyashenko, Ordinary Differential Equations, Encyclopaedia of Math. Sci. 1, Dynamical Systems 1, Springer-Verlag, 1986. MR 89g:58060

[2] A. Bruno, Local Methods in Nonlinear Differential Equations, Springer-Verlag, 1989. MR 90c:58150

[3] K.T. Chen, Equivalence and decomposition of vector fields about an elementary critical point, Amer. J. Math., 85 (1963), 693-722. MR 28:3224

[4] Ph. Hartman, On local homeomorphisms of Eucliden spaces, Bol. Soc. Mat. Mexicana., 5 (1960), 220-241. MR 25:5253

[5] F. Ichikawa, Finitely determined singularities of formal vector fields, Invent. Math., 66 (1982), 199-214. MR 83j:58021

[6] S. Sternberg, On the structure of local homeomorphisms of Euclidean n-space. II, Amer. J. Math., 80 (1958), 623-631. MR 20:3336

[7] F. Takens, Normal forms for certain singularities of vector fields, Ann. Inst. Fourier, 23 No. 2 (1973), 163-195. MR 51:1872

[8] J. Yang, Polynomial Normal Forms for Vector Fields on $\mathbf{R}^{3}$, Duke Math. J., 106 No. 1 (2001), 1-18. MR 2001m:34165

Mam, Institute of Mathematics, Peking University, Beijing, 100871, People's RepubLIC OF CHINA

E-mail address: yang@sxx0.math.pku.edu.cn

E-mail address: jyang@math.pku.edu.cn 